\title{
Zur fraglichen Identität des Münzmeisters Q. Marcius (RRC 283)
}

Manfredi Zanin, Venezia

Abstract: This article discusses the identity of the moneyer Q. Marcius (RRC 283 - ca. 118 $\mathrm{BCE}$ ). Many scholars renounced any attempt to identify him, but some evidence suggests that the moneyer could have been the son of Q. Marcius Rex (cos. 118), who died during his father's consulate. Although we cannot completely exclude the possibility that the moneyer was a member of a family still lacking a cognomen, our identification represents a plausible solution.

Keywords: Q. Marcius Rex, Marcii, Münzmeister, triumviri monetales, Prosopographie, Cognomen.

Ungefähr 118-117 v. Chr., folgt man M.H. Crawfords Chronologie, ${ }^{1}$ liess das amtierende Kollegium der triumviri monetales eine Denarserie prägen, auf deren Avers und Revers der bekannte Kopf der Roma bzw. eine wagenfahrende Siegesgöttin und die abgekürzten Namen der drei verantwortlichen Magistrate zu finden sind. Zwei Münzmeister sind kaum identifizierbar, da nur die ersten Buchstaben des jeweiligen Praenomens und Gentiliciums eingeprägt wurden $(C \cdot F$ und $L \cdot R$ ). Der dritte Name, Q·MAR, kann dagegen mühelos mit Q. Marcius aufgelöst werden.

Strittig ist in der Forschung die Identität dieses Münzmeisters. Manche halten es für ausgeschlossen, diese Person zu identifizieren. ${ }^{2}$ Andere haben verschiedene Vorschläge zu seiner Identifikation in die Diskussion eingebracht: E. Babelon meinte, dass Q. Marcius ein unbekannter Sohn des 193 v. Chr. gefallenen gleichnamigen Militärtribuns gewesen sei; H.A. Grueber hat ihn dagegen mit dem vermutlichen Sohn eines Q. Marcius Libo - ebenfalls Münzmeister ca. 30 Jahre zuvor ${ }^{3}$ - identifiziert. $^{4}$ In jüngerer Zeit ist man eher skeptisch, um Crawford zu zitieren: «The [...] moneyer, even if a Q. Marcius, is virtually unidentifiable».5 Dennoch soll im Folgenden die prosopographische Frage erneut geprüft werden.

Die Tatsache, dass der Münzmeister sich mit der Signatur Q·MAR erkennbar machen wollte, legt die Vermutung nahe, er sei Mitglied entweder einer (noch) cog-

\footnotetext{
* Für sprachliche Korrekturen und Verbesserungen des vorliegenden Beitrages bin ich Herrn Prof. Dr. Stefan Rebenich zu grossem Dank verpflichtet.

$1 \quad$ M.H. Crawford, Roman Republican Coinage (Cambridge 1974) (folgend als RRC abgekürzt) 299300, Nr. 283.

$2 \quad$ Vgl. Th. Mommsen, Geschichte des römischen Münzwesens (Berlin 1860) 524, Nr. 108, Anm. 194; Fr. Münzer, «Marcius $30 », R E$ 14,2 (1930) 1546; RRC 299-300, Nr. 283; angeblich auch E.A. Sydenham, The Coinage of the Roman Republic (London 1952) 70, Anm. 541.

$3 \quad$ RRC 255, Nr. 215; Signatur: Q·MARC LIB (ca. 148 v. Chr).

$4 \quad$ E. Babelon, Monnaies de la République Romaine II (Paris 1886) 189; H.A. Grueber, Coins of the Roman Republic in the British Museum II (London 1910) 256, Anm. 1.

$5 \quad R R C 300$.
} 
nomenlosen, wohl nicht der nobilitas zugehörigen Familie gewesen oder aber eines derart berühmten Geschlechtes, dass es ihm nicht notwendig schien, die Prägung auch mit dem Cognomen zu signieren. Zugunsten der ersten Annahme spricht, dass die anderen, zur gens Marcia gehörenden Münzmeister ihre Prägungen eher mit dem Cognomen als mit dem Gentilicium signierten. ${ }^{\mathbf{6}}$ Trotzdem muss hervorgehoben werden, dass weitere mit dem Cognomen signierende Münzmeister des marcischen Geschlechts erst in späteren Jahren hervortreten; die einzigen Ausnahmen sind der oben erwähnte Q. Marcius Libo - der eben aufgrund seiner novitas das Bedürfnis gehabt haben dürfte, sein Cognomen zur Schau zu stellen, um sich von den adligen Q. Marcii zu unterscheiden - und Q. Marcius Philippus (ca. 129 v. Chr), ${ }^{7}$ der im selben Zeitraum, in dem auch Marcii anderer Familien auf der politischen Bühne agierten, die Münzmeisterschaft bekleidete, sodass die Nennung des Cognomens nicht nur der Selbstdarstellung diente, sondern den Münzmeister auch eindeutig identifizieren sollte.

Geht man umgekehrt davon aus, dass unser Q. Marcius hinreichend bekannt gewesen wäre, müsste dieser Münzmeister in den drei adligen Zweigen der gens gesucht werden: den Marcii Censorini, den Marcii Philippi und den Marcii Reges. ${ }^{\mathbf{8}}$ Dass Q. Marcius ein Censorinus war, ist mit Bestimmtheit auszuschliessen: Die bekannten Marcii Censorini trugen ausschliesslich die Praenomina Gaius, Gnaeus und Lucius. ${ }^{9}$ Das Praenomen Quintus ist dagegen in den zwei übrigen Familien der nobiles Marcii verbreitet. Von den Philippi sind indessen ausnahmslos Münzmeister bezeugt, die das Cognomen Philippus zur aristokratischen Selbststilisie-

6 Zur Entwicklung und Anwendung des Cognomens in der römisch-republikanischen Führungsschicht, namentlich im Hinblick auf seine Verbreitung unter den Familien von equites und homines novi, vgl. noch $\mathrm{Cl}$. Nicolet, "L'onomastique des groupes dirigeants sous la république», in L'onomastique latine (Paris 1977) 45-61, bes. 53-56.

$7 \quad$ RRC 284-285, Nr. 259 (Signatur: Q·PILIPVS). Der Münzmeister - Vater des späteren Konsuls 91 v.Chr., L. Marcius Philippus - ist etwa mit dem von Cicero (Balb. 11,28) erwähnten Q. Philippus zu identifizieren, der ins Exil ging und Bürger Nucerias wurde. Vgl. dazu Fr. Münzer, «Marcius 81-82», RE 14,2 (1930) 1579; J. van Ooteghem, Lucius Marcius Philippus et sa famille (Bruxelles 1961) 99-100; E.S. Gruen, «Political Prosecutions in the 90’s B.C.», Historia 15 (1966) 32-64 (hier 62-63); E.S. Gruen, Roman Politics and the Criminal Courts, 149-78 B.C. (Cambridge, MA 1968) 210-211; G.P. Kelly, A History of Exile in the Roman Republic (New York 2006) 79, 169-170; K. Zmeskal, Adfinitas. Die Verwandschaften der senatorischen Führungsschicht der römischen Republik von 218-31 v. Chr. (Passau 2009) 183, 391.

$8 \quad$ Zur mythhistorischen Tradition und numismatischen Selbstdarstellung der einzelnen marcischen Familien vgl. jetzt K.-J. Hölkeskamp, «Im Gewebe der Geschichte(n). Memoria, Monumente und ihre mythhistorische Vernetzung", Klio 94,2 (2012) 380-414, wieder in: K.-J. Hölkeskamp, Libera res publica. Die politische Kultur des antiken Rom - Positionen und Perspektiven (Stuttgart 2017) 237271, hier bes. 249-257.

9 Vgl. K. Zmeskal, a.O. (Anm. 7) 181-182. Übrigens signierten die Censorini ihre Münzen mit Praenomen und Cognomen; vgl. RRC 357-361, Nr. 346; 374-375, Nr. 360; 377-378, Nr. 363: C.CENSORIN (ca. 88/87 v.Chr.) und L·CENSOR (82 v. Chr.). 
rung auf ihre Prägungen setzen liessen. ${ }^{10} \mathrm{Q}$. Marcius Philippus wiederum bekleidete, wie oben schon ausgeführt, die Münzmeisterschaft kurz vor unserem Q. Marcius. Alles spricht folglich gegen eine Zuweisung des Letztgenannten in die gens der Marcii Philippi.

Es bleibt der Zweig der Marcii Reges. Kein dieser Familie zugehörender Münzmeister ist indes eindeutig bezeugt, und folglich ist die Praxis ihrer Selbstdarstellung auf Münzen - namentlich die repräsentative Anwendung von Praenomina, Gentilicium und Cognomen - nicht bekannt. Betrachtet man andererseits die Namen und die Biographien der Marcii Reges, stösst man auf ein sehr interessantes Ereignis ihrer Familiengeschichte. Q. Marcius Rex bekleidete 118 v. Chr. das Konsulat, ${ }^{11}$ erlitt aber im selben Jahre einen tragischen Verlust, wie Valerius Maximus $^{12}$ in einer Passage der Facta et dicta memorabilia berichtet:

Q. Marcius Rex, superioris Catonis ${ }^{13}$ in consulatu collega, filium summae pietatis et magnae spei et, quae non parva calamitatis accessio fuit, unicum amisit, cumque se obitu eius subrutum et eversum videret, ita dolorem altitudine consilii coercuit, ut a rogo iuvenis protinus curiam peteret senatumque, quem eo die lege habere oportebat, convocaret. Quod nisi fortiter maerorem ferre scisset, unius diei lucem inter calamitosum patrem et strenuum consulem neutra in parte cessato officio partiri non potuisset.

«Q. Marcius Rex, Amtsgenosse des älteren Cato im Konsulat, verlor seinen äußerst pflichtbewussten, vielversprechenden und - was den Verlust nicht wenig verschlimmerte - einzigen Sohn; und obwohl ihm dessen Tod einen schweren Schlag versetzte und er sich am Boden zerstört fühlte, wusste er dennoch seinen Schmerz mit derartiger Selbstbeherrschung zu zügeln, dass er sich unmittelbar nach der Feuerbestattung des jungen Mannes in die Kurie begab und den Senat zusammenrief, dessen Versammlung an diesem Tag gesetzlich vorgeschrieben war. Hätte er seine Trauer nicht tapfer ertragen können, wäre er nicht fähig gewesen, an diesem Tag zwischen dem schmerzlich betrübten Vater und dem tüchtigen Konsul zu unterscheiden, ohne zu vergessen, welche Pflichten er beiden Rollen schuldete.»

Hier interessiert nicht das exemplum virtutis des Q. Marcius Rex, sondern die Nachricht, dass sein einziger Sohn, dem eine glänzende Karriere vorgezeichnet war (magnae spei), im Jahre seines Konsulats umkam. Es ist verlockend, diesen jung gestorbenen Marcius Rex (gewiss ebenfalls ein Quintus) mit dem Münzmeister Q. Marcius zu identifizieren. Wie eingangs erwähnt, datiert Crawford diese Prägung in die Jahre 118-117 v.Chr. Akzeptiert man die vorgeschlagene Identifi-

10 Vgl. Anm. 7 und RRC 307-308, Nr. 293; 448, Nr. 425: L·PHILIPPVS (ca. 113/112 v. Chr) und PHILIPPVS (ca. 56 v. Chr.). Zum Cognomen Philippus vgl. auch J. Briscoe, «The cognomen Philippus», Gérion 2 (1984) 151-153.

11 Vgl. MRR I, 527.

12 Val. Max. 5,10,3.

13 Zur Verwechslung zwischen Cato dem Älteren und dessen Sohn vgl. R. Combès (hrsg.), Valère Maxime. Faits et dits mémorables II (Paris 1997) 137, Anm. 3. 
kation mit Q. Marcius Rex dem Jüngeren, könnte seine Münzmeisterschaft auf das Jahr 118 v. Chr. datiert werden; andererseits könnte dieses Jahr als terminus ante quem gelten, sodass es durchaus denkbar wäre, dass er vor seinem Tod noch ein weiteres Amt innerhalb des cursus honorum (etwa die Quästur ${ }^{\mathbf{1 4}}$ ) bekleidet hatte. $^{15}$

Gegen diese Interpretation könnte man einwenden, dass eine Signatur mit dem Cognomen Rex viel eingängiger als eine mit dem blossen Gentilicium gewesen wäre: Die Münzmeister der gentes nobiles bedienten sich meistens des jeweiligen Cognomens (direkt oder indirekt ${ }^{16}$ ). Trotzdem fehlt es nicht an Fällen, in denen Münzmeister aus der Nobilität (namentlich Mitglieder der Cassii Longini ${ }^{\mathbf{1 7}}$, der Domitii Ahenobarbi ${ }^{18}$ und vielleicht auch der Sulpicii Galbae ${ }^{\mathbf{1 9}}$ ) allein Praenomen und Gentilicium anführten. Eine solche Praxis ist auch für die Marcii Reges nicht a priori zu verneinen.

\begin{abstract}
14 Vgl. aber unten.
15 Es kommt häufiger vor, dass in den Jahren, in denen Mitglieder einer Familie die Münzmeisterschaft bekleideten, andere Angehörige derselben Familie das Konsulat, die Prätur usw. innehatten. Vgl. dazu A.M. Burnett, «The Authority to Coin in the Late Republic and Early Empire», NumChron 17 (1977) 37-63, obschon seine Hypothese, die Münzmeister seien von den Konsuln ernannt worden, wohl abzuweisen ist: H.B. Mattingly, «The Management of the Roman Republican Mint», AnnIstItNum 29 (1982) 9-46, wieder in H.B. Mattingly, From Coins to History. Selected Numismatic Studies (Ann Arbor 2004) 227-259, hier 228-229; M.H. Crawford, Coinage and Money under the Roman Republic (London 1985) 56 Anm. 6; D.B. Hollander, «The Management of the Mint in the Late Roman Republic», AncHistB 13 (1999) 14-27; Burnett selbst hat seine Meinung geändert: A.M. Burnett, Coinage in the Roman World (London 1987) 17. Zum Zusammentreffen zwischen Amtsjahren der Konsuln und der Münzmeister vgl. auch Hollander, a.O. 15; man sollte in diesem Zusammenhang auch die Amtsjahre von Prätoren und Zensoren betrachten, denn diese Koinzidenzen sind eher auf den allgemeinen Einfluss der jeweiligen Familie als auf eine punktuelle Einflussnahme zurück-
\end{abstract} zuführen.

16 Als Beispiele für den indirekten Hinweis auf das Cognomen vgl. die anonyme Prägung mit Elefantenkopf für L. Caecilius Metellus, wahrscheinlich Diadematus (RRC 287, Nr. 262; ca. 128 v.Chr.), oder den Eselskopf für M. Iunius Silanus (RRC 259, Nr. 220; ca. 145 v.Chr., M.IVNI).

$17 R R C$ 290, Nr. 266 (C. Cassius Longinus, ca. 126 v.Chr., C.CASSI); 370-371, Nr. 355 (C. Cassius Longinus, ca. 84 v. Chr., C.CASSIVS); 403, Nr. 386 (L. Cassius Q.f. Longinus, ca. 78 v. Chr., L.CASSI.Q.F); 452, Nr. 428 (Q. Cassius Longinus, ca. 55 v.Chr., Q.CASSIVS); vgl. aber RRC 440, Nr. 413 (L. Cassius Longinus, ca. 63 v.Chr.), Signatur: LONGIN und auf dem Avers auftauchende Kontrollbuchstaben, die in der richtigen Reihefolge (LCAS2I) das Praenomen und das Gentilicium des Münzmeisters wiedergeben.

18 RRC 218, Nr. 147 (Cn. Domitius Ahenobarbus, ca. 189-180 v.Chr., CN·DOM) 286, Nr. 261 (Cn. Domitius, ca. 128 v.Chr., wahrscheinlich ein Ahenobarbus - CN·DOMIT), 298-301, Nr. 282, 285 (Cn. Domitius Ahenobarbus, ca. 118/116-115 v.Chr., CN·DOM/CN·DOMI). Das Cognomen Ahenobarbus kommt erst um 40 v. Chr. auf den imperatorischen Prägungen des Cn. Domitius Ahenobarbus, des späteren Konsuls von 32 v. Chr., vor: RRC 527-528, Nr. 519, 521.

$19 R R C$ 320, Nr. 312, C. Sulpicius C. f., ca. 106 v.Chr. - C.SVLPICI.C.F, der meistens als ein Galba betrachtet wird. Es ist aber zu beachten, dass ein P. Sulpicius Galba, kurulischer Ädil ca. 69 v. Chr., auf seine Münzen Praenomen und Cognomen setzen liess (RRC 418, Nr. 406: P.GALB AED CVR), während ein Ser. Sulpicius Rufus, Münzmeister ca. 51, mit Praenomen und Gentilicium signierte (RRC 459-460, Nr. 438: SER·SVLP). 
Auch wenn wir nicht völlig ausschliessen können, dass der Münzmeister Q. Marcius zu einer cognomenlosen Familie gehörte, ${ }^{20}$ sprechen unsere Überlegungen für die Annahme, dass Q. Marcius der Sohn des Konsulars Q. Marcius Rex gewesen ist.

Als letzter Punkt ist das vermutliche Todesalter des jüngeren Q. Marcius Rex zu erwägen. Das Geburtsdatum des Konsuls von 118 v.Chr. muss mindestens ins Jahr 161 v.Chr. fallen; jenes seines Vaters, Prätors von 144 v.Chr., ins Jahr 184 v.Chr. oder kurz zuvor. Q. Marcius Rex, Konsul von 118 v.Chr., muss wiederum etwa zwischen 20 und 25 Jahre alt gewesen sein, als sein Sohn geboren wurde. Angesichts der einzelnen geschätzten Zeitabstände scheint es berechtigt, das Geburtsdatum des jüngeren Marcius Rex ungefähr ins Jahr 140 v. Chr. zu setzen: Demzufolge wäre er im Alter von ca. 22 Jahren gestorben; damit kann wahrscheinlich ausgeschlossen werden, dass er die Quästur erreichte. ${ }^{21}$

Korrespondenz:

Manfredi Zanin

Università Ca’ Foscari Venezia

Dipartimento di Studi Umanistici

Dorsoduro 3484/D, Calle Contarini

I-30123 Venezia

manfredi.zanin@unive.it

20 So will etwa H. Zehnacker, Moneta. Recherches sur l'organisation e l'art des émissions monétaires de la république romaine (Rome 1973) 851, 929 Anm. 1, 930 Anm. 5 die Prägung aus rein stilistischen Gründen eher in das Jahrzehnt 110-100 v. Chr. datieren.

21 Es ist unklar, ob Q. Marcius Rex, Konsul 68 v.Chr., ein Zweitgeborener oder ein Enkel des Konsuls von 118 v. Chr. war. Angesichts seines wohl kurz vor dem Jahr 110 anzusetzenden Geburtsdatums und der hier vorgeschlagenen chronologischen Rekonstruktion erscheint es schwierig, eine Filiation des Konsuls von 68 v. Chr. von dem jung gestorbenen Q. Marcius Rex zu postulieren. Dazu vgl. Fr. Münzer, «Marcius 92», RE 14,2 (1930) 1583-1586, bes. 1583; K. Zmeskal, a.O. (Anm. 7). 